\title{
A PAPER ON PERFORMANCE ANALYSIS OF MICROSTRIP RECTANGULAR PATCH ANTENNA
}

Vineet Chhabra, Shambhu Dayal sharma

E-Mail Id: vineet.chhabra@anandice.ac.in, shambhu.sharma@anandice.ac.in Department of Electronics \& Communication Engineering, Anand International College of Engineering Jaipur (Rajasthan), India

Abstract-The study of micro strip patch antennas has made great progress in recent years. Compared with conventional antennas, micro strip patch antennas have more advantages and better prospects. They are lighter in weight, low volume, low cost, low profile, smaller in dimension and ease of fabrication and conformity. Moreover, the micro strip patch antennas can provide dual and circular polarizations, dual-frequency operation, frequency agility, broad band-width, feed line flexibility, beam scanning omnidirectional patterning. In this paper we discuss the microstrip antenna, types of microstrip antenna, feeding techniques and application of microstrip patch antenna with their advantage and disadvantages over conventional microwave antennas. In this paper we will try to find the changes in the output of the Microstrip antenna when we change the input values. Analyzing rectangular Line-Fed Microstrip antenna using PCAAD 6.0 software.

Keywords: Directivity, Efficiency, 3 db Beam width.

\section{INTRODUCTION}

Over the recent past, we have witnessed a considerable amount of growth in wireless industry both in terms of mobile technology and subscribers both network operators and vendors have realized the importance of efficient network with equally efficient design processes. Wireless communications have become pervasive. with all the technological advances Antenna efficiency has a important role in the wireless technology in this paper we will try to identify the different factors affecting the antenna parameters so that any change may be utilizes for the future research in wireless communication. Antenna is a transducer designed to transmit or receive electromagnetic waves. Microstrip antennas have several advantages over conventional microwave antenna and therefore are widely used in many practical applications[1].

\section{METHODS}

\subsection{Overview of Patch Antenna}

Microstrip or patch antennas are becoming increasingly useful because they can be printed directly onto a circuit board. Microstrip antennas are becoming very widespread within the mobile phone market. Patch antennas are low-cost, have a low profile and are easily fabricated [2]. Consider the microstrip antenna shown in Figure 1, fed by a microstrip transmission line. The patch antenna, microstrip transmission line and ground plane are made of high conductivity metal (typically copper). The patch is of length L, width W, and sitting on top of a substrate (some dielectric circuit board) of thickness h with permittivity or dielectric constant. The thickness of the ground plane or of the microstrip is not critically important. Typically the height $\mathrm{h}$ is much smaller than the wavelength of operation, but should not be much smaller than 0.025 of a wavelength (1/40th of a wavelength) or the antenna efficiency will be degraded.

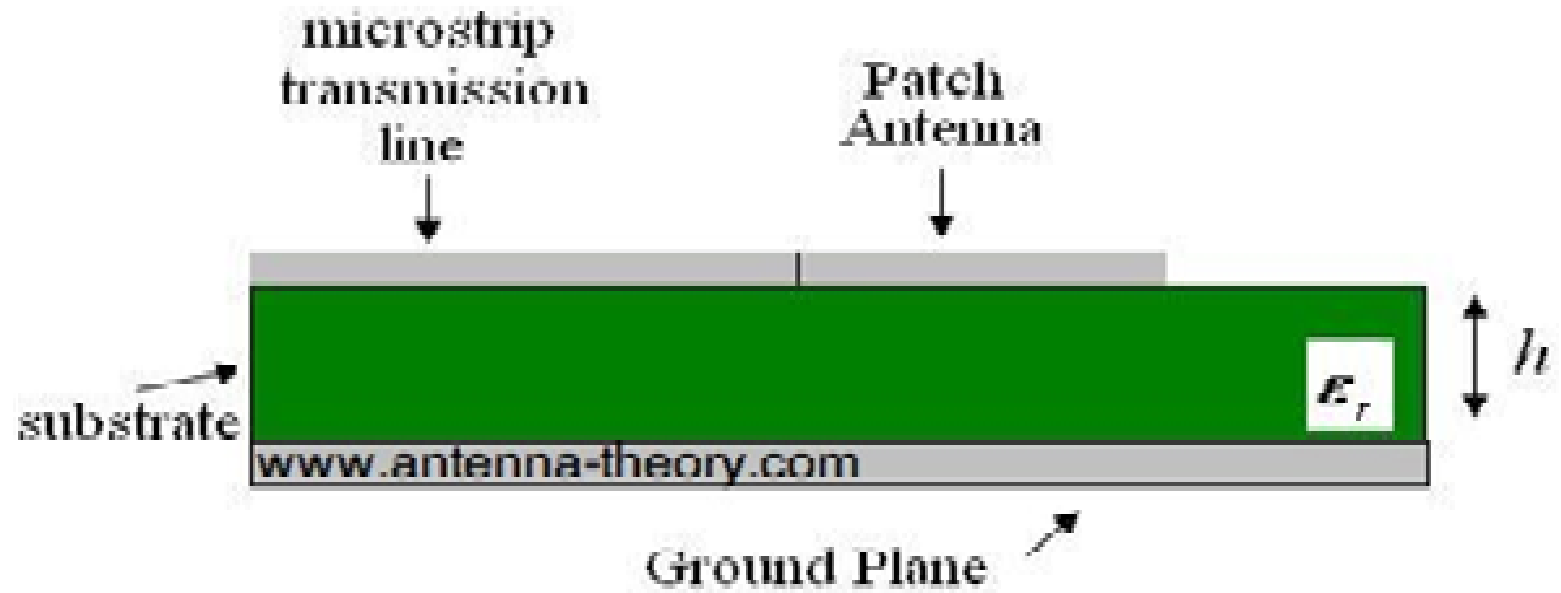

Fig. 2.1 Geometry of Microstrip (Patch) Antenna

Consider a square patch antenna fed at the end as before in Figure 1. Assume the substrate is air (or Styrofoam, with a permittivity equal to 1 ), and that $\mathrm{L}=\mathrm{W}=1.5$ meters, so that the patch is to resonate at $100 \mathrm{MHz}$. The height $\mathrm{h}$ is taken to be $3 \mathrm{~cm}$. Note that micro strips are usually made for higher frequencies, so that they are much smaller in practice. When matched to a $200 \mathrm{Ohm}$ load, the magnitude of S11 is

DOI Number: https://doi.org/10.30780/specialissue-ICRDET-2019/006

pg. 35 


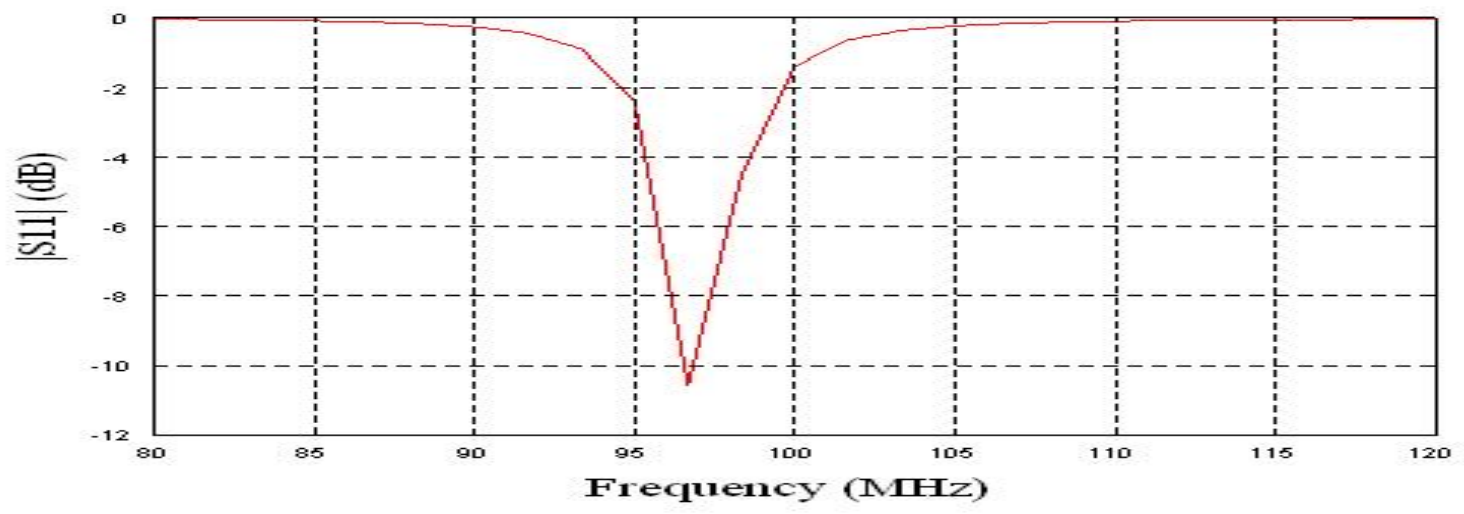

Fig. 2.2 Magnitude of S11 versus Frequency for Square Patch Antenna

\section{FEEDING TECHNIQUE}

A feed line is used to excite to radiate by direct or indirect contact. There are many different techniques of feeding and four most popular techniques are coaxial probe feed, micro strip line, aperture coupling and proximity coupling[3] . Coaxial probe feeding is feeding method in which that the inner conductor of the coaxial is attached to the radiation patch of the antenna while the outer conductor is connected to the ground plane. Advantages of coaxial feeding is easy of fabrication, easy to match, low spurious radiation and its disadvantages is narrow bandwidth, Difficult to model specially for thick substrate.

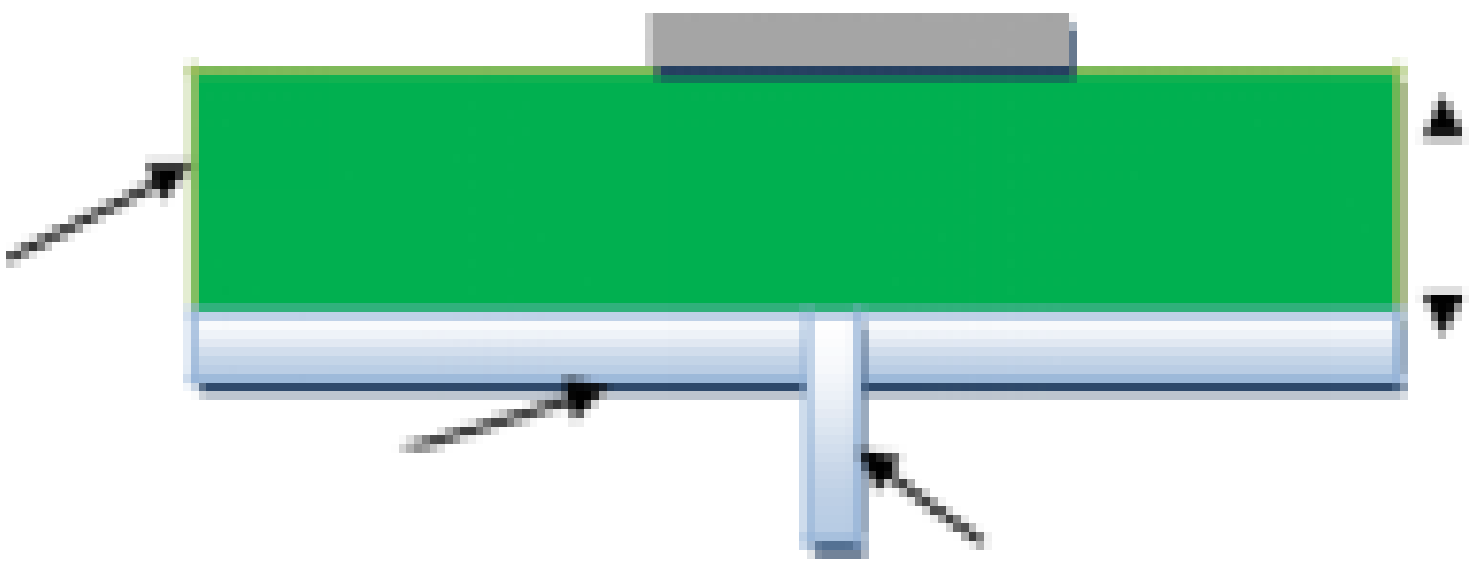

Fig. 3.1 Coaxial Probe Feed Patch Antenna

The Micro strip patch antennas are well known for their performance and their robust design, fabrication and their extent usage[4].The advantages of this Micro strip patch antenna are to overcome their d-merits such as easy to design, light weight etc., the applications are in the various fields such as in the medical applications, satellites and of course even in the military systems just like in the rockets, air-crafts missiles etc. the usage of the Micro strip antennas are spreading widely in all the fields and areas and now they are booming in the commercial aspects due to their low cost of the substrate material and the fabrication. It is also expected that due to the increasing usage of the patch antennas in the wide range this could take over the usage of the conventional antennas for the maximum applications.

\section{SIMULATION AND RESULT}

This routine analyzes a rectangular microstrip line fed microstrip antenna using the transmission line enter the patch length ,patch width ,Dielectric constant ,loss tangent as shown in the tables below and PCADD will compute bandwidth, efficiency,directivity in $\mathrm{db}$ so we have taken the following cases in PCADD Software and got the different results and radiation pattern. In the following result we kept Dielectric constant 2.2, substrate thickness $0.079 \mathrm{~cm}$ and loss tangent 0.001 .

Case A: Patch Length $=3.315 \mathrm{~cm}$, Patch Width $=3.317 \mathrm{~cm}$

\begin{tabular}{|l|l|}
\hline Quantity & PCADD 6.0 Measured \\
\hline Bandwidth & $4.2 \%$ \\
\hline efficiency & $68.7 \%$ \\
\hline Directivity db & 5.8 \\
\hline
\end{tabular}


ICRDET-2019, September 14-15, 2019, AICE, Jaipur, India

International Journal of Technical Research \& Science (Special Issue) ISSN No.:2454-2024 (online)

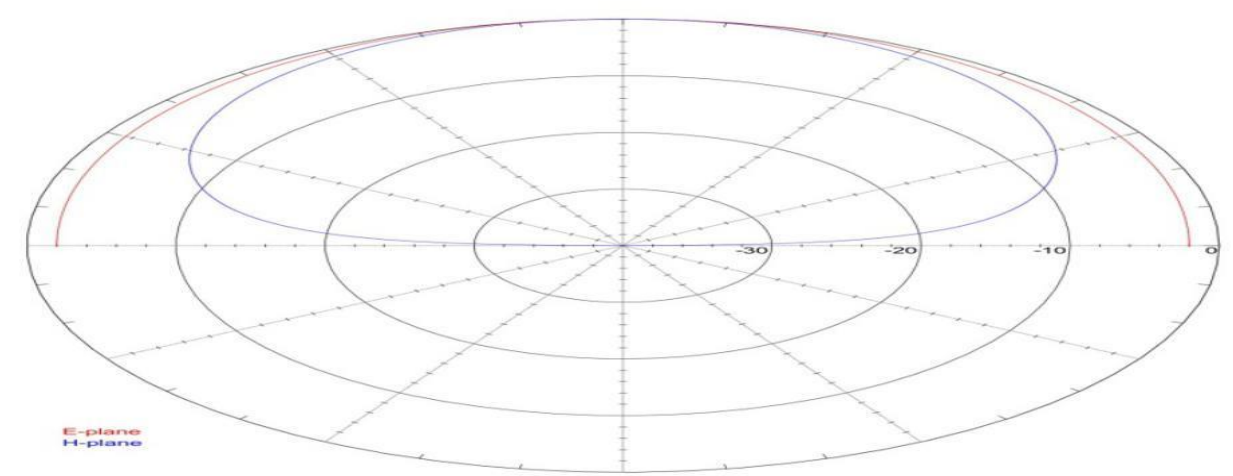

Fig 4.1 Radiation Pattern Case A

Case B: Patch Length $=5.315 \mathrm{~cm}$, Patch Width $=5.317 \mathrm{~cm}$

\begin{tabular}{|l|l|}
\hline Quantity & PCADD 6.0 Measured \\
\hline Bandwidth & $0.8 \%$ \\
\hline effeciency & $66.0 \%$ \\
\hline Directivity db & 7.3 \\
\hline
\end{tabular}

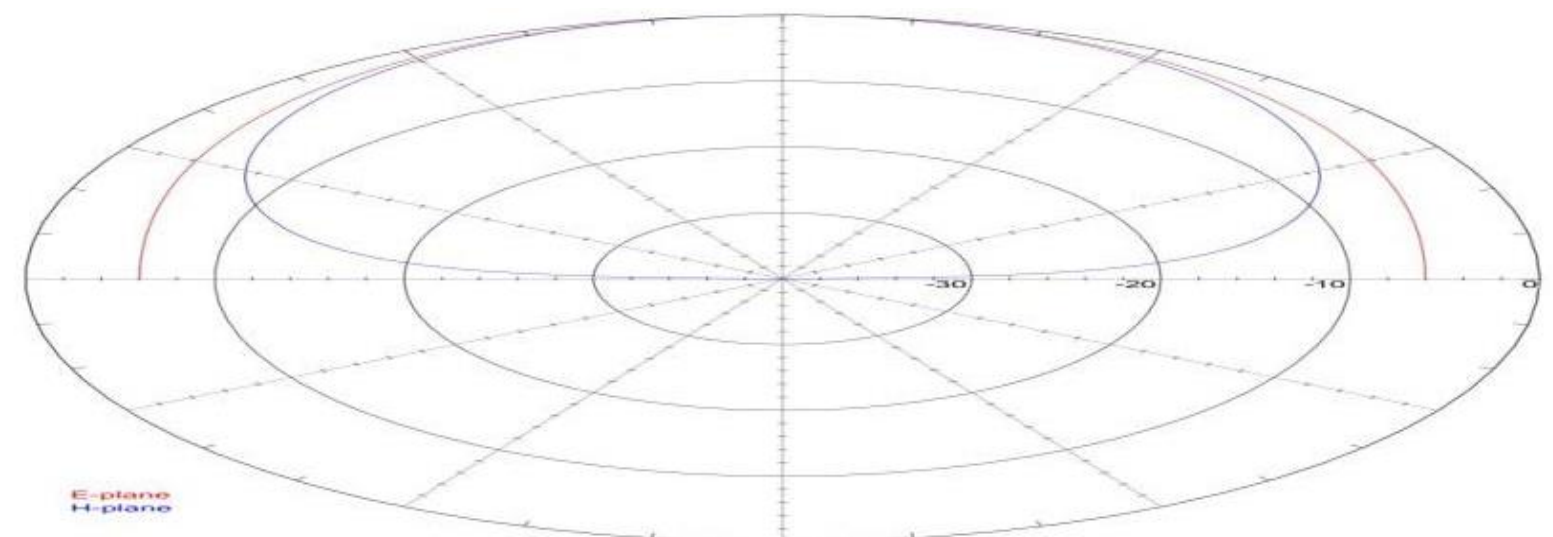

Fig. 4.2 Radiation Pattern Case B

Case C: Patch Length $=7.315 \mathrm{~cm}$,Patch Width $=7.317 \mathrm{~cm}$

\begin{tabular}{|l|l|}
\hline Quantity & PCADD6.0 Measured \\
\hline Bandwidth & $4.6 \%$ \\
\hline efficiency & $52.6 \%$ \\
\hline Directivity db & 9.2 \\
\hline
\end{tabular}

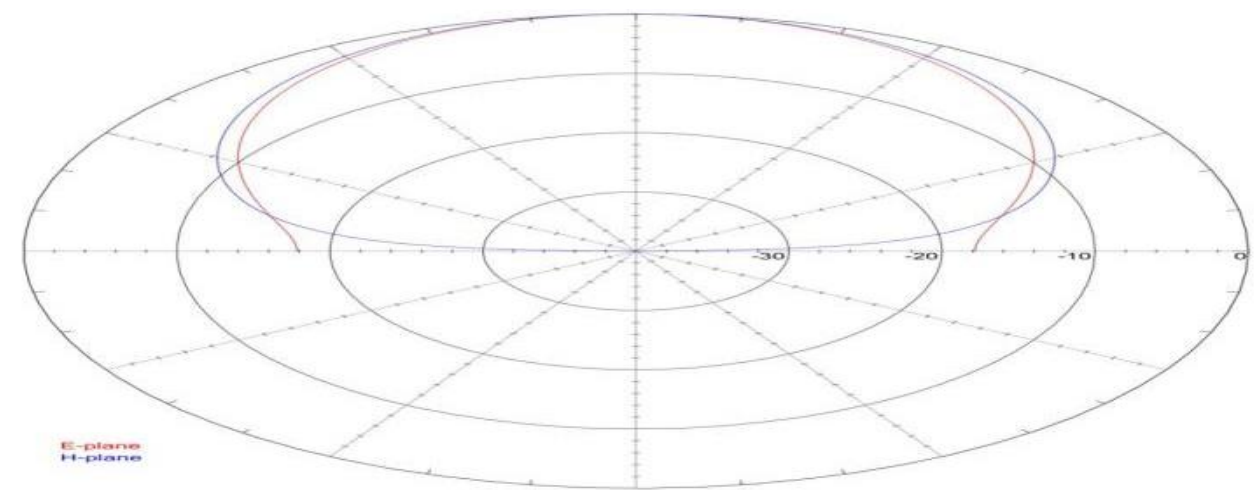

Fig. 4.3 Radiation Pattern Case C

DOI Number: https://doi.org/10.30780/specialissue-ICRDET-2019/006 


\section{CONCLUSION}

Above simulated results are quite clearly showing that by increasing the patch width and length directivity of an antenna is improved. As we know Directivity is a fundamental antenna parameter. It is a measure of how 'directional' an antenna's radiation pattern is. An antenna that radiates equally in all directions would have effectively zero directionality, and the directivity of this type of antenna would be 1 (or $0 \mathrm{~dB}$ ) So by this we clearly say that despite using antenna array to increase the directivity we can clearly see that slight change in antenna parameters we can increase the directivity of Patch Antenna.

\section{REFERENCES}

[1] James j., and P.S. Hall (Eds), Handbook of microstrip antenna, Peter Peregrinus, London, UK, 1989.

[2] Ramesh Garg, Prakash Bartia, Inder Bahl, Apisak Ittipiboon, '’Microstrip Antenna Design Handbook', 2001, pp 1-68, 253-316

[3] Robert A. Sainati, CAD of Microstrip Antennas for Wireless Applications, Artech House Inc, Norwood, MA, 1996.

[4] C.A. Balanis, Antenna theory: analysis and design, 2nd ed., John Willey and \& Son, Inc., 1997. 